\title{
MEASURING MASS LOSS RATES FROM GALACTIC SATELLITES
}

\author{
KATHRYN V. JOHNSTON \\ Institute for Advanced Study, \\ Olden Lane, Princeton, NJ 08540, USA \\ STEINN SIGURDSSON \\ Institute of Astronomy, Madingley Road, Cambridge, CB3 OHA \\ and \\ LARS HERNQUIST \\ Board of Studies in Astronomy and Astrophysics, \\ University of California, Santa Cruz, CA 95064
}

Number count profiles of many Galactic and some extra-galactic satellite systems show evidence for associated stars beyond the cut-off in density that is identified as the point of tidal limitation (e.g. Irwin \& Hatzidimitriou 1995, Grillmair et al. 1995). These "extra-tidal" stars are assumed to be debris lost from the satellite due to heating or stripping by the Galactic tidal field or (in the case of globular clusters) evaporation of stars over the tidal boundary. In this contribution we present a method for using these features to measure the mass loss rate from the satellite, and test it on the results of numerical simulations of satellite disruption. A more detailed discussion of all aspects of this work can be found in Johnston, Sigurdsson \& Hemquist (1998).

In the numerical simulations, the satellite's evolution along an orbit in a three component rigid model of the Galaxy is followed using a self-consistent field (SCF) code (developed by Hernquist \& Ostriker 1992) to calculate the mutual interactions of stars in the satellite. Figure 1 shows the annularly averaged number surface density from one example simulation "observed" from the viewpoint of the center of the Galaxy after several Gigayears. The closed (open) symbols show the profile recovered if only bound (all) stars are considered. Clearly there is a break in the open symbols at the radius $r_{\text {break }}$ where the analysis becomes dominated by unbound stars.

Tremaine (1993) pointed out that the change in the orbital frequency of a star torn from the satellite at $r_{\text {break }}$ should approximately be given by $\Delta \Omega \sim r_{\text {break }} d \Omega / d R$, where $\Omega(R)$ is the frequency of a circular orbit in the parent galaxy at radius $R$. Hence, debris will spread over an angular distance comparable to the size of the cluster $\left(r_{\text {break }} / R\right)$ in a time $\left(r_{\text {break }} / R\right) / 2 \Delta \Omega \sim T_{\text {orb }} / \pi$, where $T_{\text {orb }}$ is the azimuthal time period of the orbit. This suggests that we can estimate the average surface density of stars in an annulus between $r_{\text {break }}$ and $r$ from the centre of the cluster to be

$$
\left\langle\Sigma_{\mathrm{xt}}(r)\right\rangle=\left[\frac{d m}{d t} \frac{\left(r-r_{\text {break }}\right)}{r_{\text {break }}} \frac{T_{\text {orb }}}{\pi}\right] /\left[\pi\left(r^{2}-r_{\text {break }}^{2}\right)\right],
$$

where $d m / d t$ is the mass loss rate from the cluster. We can differentiate equation (1) to find an approximate expression for the absolute surface density. This estimate 


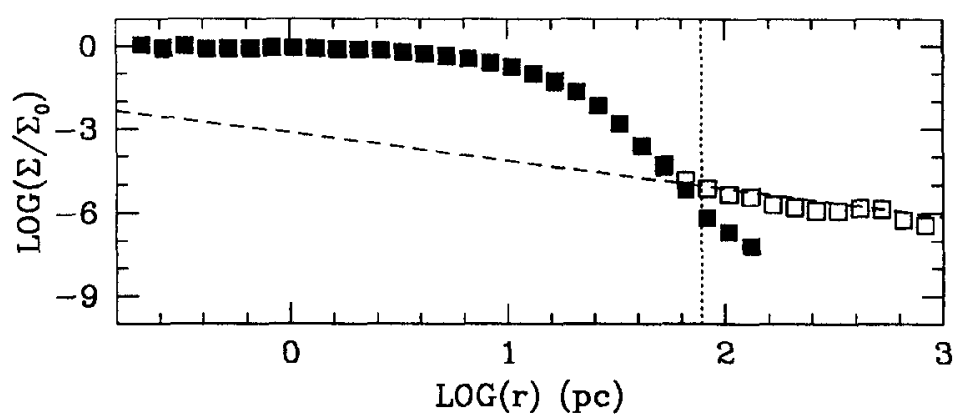

Fig. 1. Number count profiles from a simulation of satellite disruption, as viewed from the center of the Galaxy. The closed (open) symbols are for the bound (total) population of stars. The dotted line shows the position of $r_{\text {break }}$ used to model the extra-tidal population. The dashed line shows the model.

is overlaid in dashed lines on the profile shown in Figure 1 using $d m / d t$ averaged over the simulation, and $r_{\text {break }}$ indicated by the vertical dotted line. This method reproduces the extra-tidal features in all simulations with similar success.

Equation (1) can be used to measure the mass loss rate from a Galactic satellite from the density of extra-tidal stars and assuming a value for $T_{\text {orb. }}$. When tested on the simulations, this method recovered the mass loss rate to within a factor of two. When applied to observations it can be used to provide constraints on dynamical models of individual satellites and to directly measure the current destruction rate of the Galactic satellite system. Tests on simulations of mass-segregated globular clusters also indicate that this mass-loss estimate can be used to place limits on the initial mass function of stars in the cluster from local and global measurements of the present day mass function (see Johnston, Sigurdsson \& Hernquist, 1998).

\section{References}

Grillmair, C.J., Freeman, K.C., Irwin, M. and Quinn, P.J.: 1995, Astron.J. 109, 2553

Hernquist, L. \& Ostriker, J.P.: 1992, Astrophys.J. 386, 375

Irwin, M. J. and Hatzidimitriou, D.: 1995, MNRAS 277, 1354

Johnston, K.V., Sigurdsson, S. and Hernquist, L.: 1998, MNRAS, in press 